\title{
A Perspective of Arrow Speed Measurement using Various Methods
}

\author{
Muhammad Fajar Hardianto, Anton Siswo Raharjo and Tito Waluyo Purboyo \\ Department of Computer Engineering, Faculty of Electrical Engineering, Telkom University, \\ Bandung, Indonesia
}

\begin{abstract}
In archery, we need the accuracy in aiming and calculating the velocity of the arrow because of there is an archery paradox phenomenon. Archer's paradox can make an arrow flight to target with an aim is not within an actual target but rather in left or right from the actual target. Because of archer's paradox, each archer must consider speeding a velocity of an arrow. In this study, we will review some of the methods which used to measure the speed of an arrow.
\end{abstract}

$\underline{\text { Key words: Arrow, chronograph, Doppler shift, high speed video, laser sensor, IMU sensor }}$

\section{INTRODUCTION}

Archery has been existing, since, ancient time, according to the discovery of archeologist, bow and arrow has been estimated around 64,000 years ago (Backwell et al., 2008). With the advancing technology, the development of archery has become a sport. In shooting, there is some factor that affects the athlete in aiming right into the target. One of them is a speed of an arrow.

With advanced technology, there is some invention have been creating for measurement of arrow's speed. Some well-known method for measuring of speed is chronograph, Doppler shift, high-speed video recording, laser-based system and IMU sensor. In this study, the writer will be introducing some of the existing methods, review some and discuss some of the factors to getting method that needs it.

\section{MATERIALS AND METHODS}

Chronograph: A chronograph is standard for measuring a type of objects such as bullets, arrow or any projectile. There is 2 type of chronograph model which is the optical chronograph and chronograph watch. Optical chronograph is based on an optical gate that places on a device. Figure 1 the device must be placed on a flight of an arrow, so, the device can capture the optical sensor that passes through the shaft. The range of equipment can measure from $6.7 \mathrm{~m} / \mathrm{sec}$ up to $2438 \mathrm{~m} / \mathrm{sec}$, the cost of each unit can cost up to $\$ 900$ with the accuracy is around $0.5 \%$. Because of using optical sensing to get the data of travel of flight the arrow, chronograph must be in the type of light that the sensor can get the precision data such as

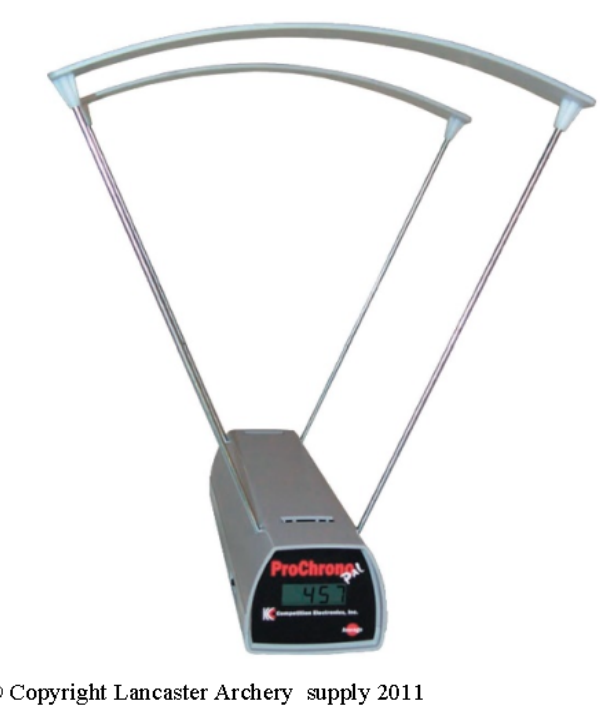

Fig. 1: Optical based chronograph

when indoor, we must using a fluorescent lamp as lightning the sensor. And because it's more like a radar system, the device only can capture one shoot at the time.

Another type of chronograph is the watch one, this is the first device that combines between watch and stopwatch to calculate the measurement of speed, travel of flight and using for NASA in Apollo mission to time a significant course correction on their perilous journey back to earth (Tao, 2006; Vroom, 1923). Figure 2 the chronograph watch works by press the start/stop button on the side of the watch to start or stop the stopwatch and push the bottom button to reset back to zero.

Corresponding Author: Muhammad Fajar Hardianto, Department of Computer Engineering, Faculty of Electrical Engineering, Telkom University, Bandung, Indonesia 


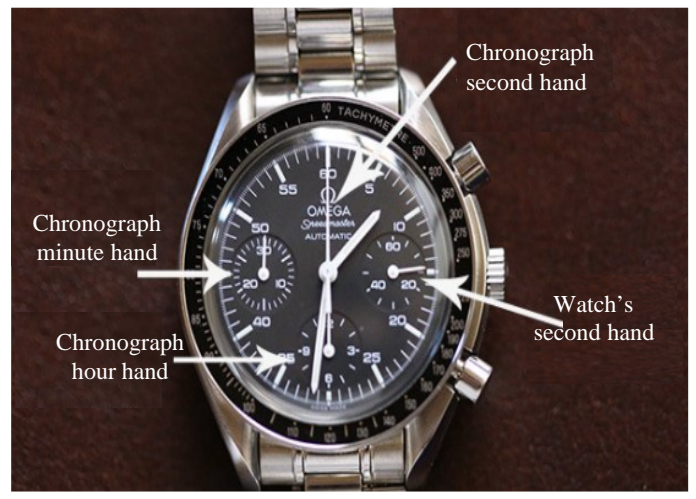

Fig. 2: Chronograph watch (Brett, 2016)

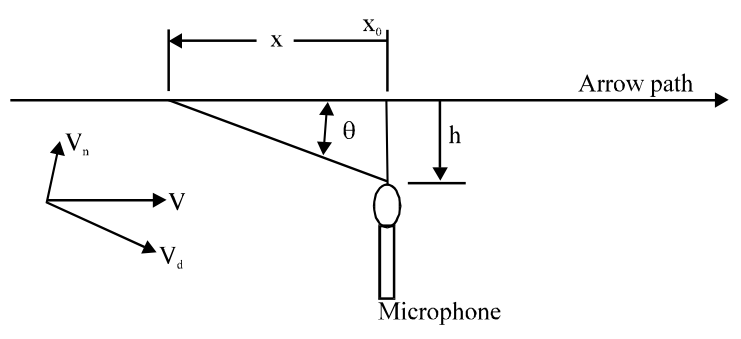

Fig. 3: Geometry of arrow (French and Kirk, 2007)

Usually, there are 3 hands: a second hand, an hour hand and a minute hand. The second hand if for or the current time is on the right-side sub-dial. When the hour hand tells about the hour of using the stopwatch and the minute hand is minutes of using the stopwatch with all parameters that we get from the chronograph it can calculate by using tachymeters.

Acoustic Doppler shift: Doppler shift is another alternative to measurement speed of an arrow using a microphone and an arrow will pass through the microphone and making the sound. With the sound of noise from the arrow is broadband because turbulence which comes from the feather in the rear of arrow (French and Kirk, 2007). By the broadband of noise, we can capture the signal of the sound and measure using Doppler-shifted formula.

Figure 3 shows the $\mathrm{V}_{-} \mathrm{d}$ is making noise through a microphone that generates a Doppler shift. There are 2 types of V_d when they are on above microphone and pass through the microphone. Note that, $\theta=90$ that cannot be ignored. When $\mathrm{V}_{-} \mathrm{d}=0$, Doppler-shifted frequency is:

$$
f=f_{s} \frac{c}{x-V d^{\prime}}
$$

Where:

$\mathrm{c}=$ The speed of sound

$\mathrm{f}_{\mathrm{s}}=$ The frequency of source formula

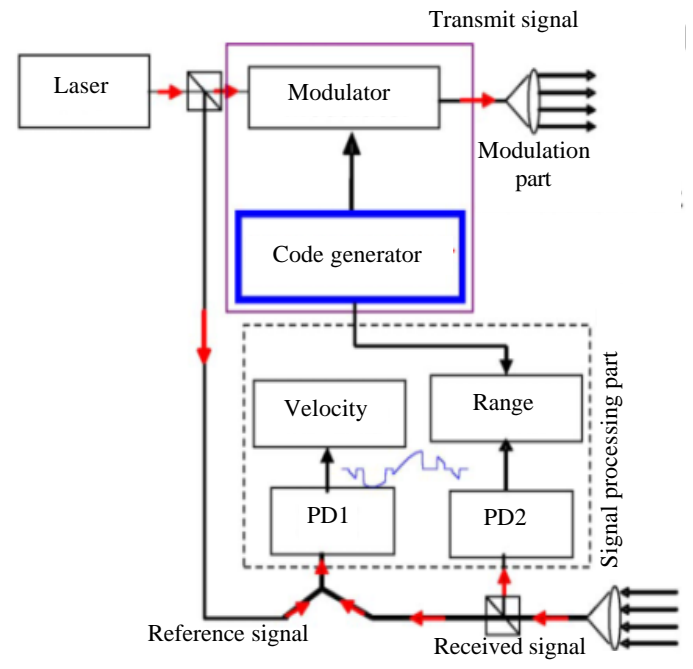

Fig. 4: Schematic of laser based Doppler shifter (Radar, 2011)

where a frequency is shifted is:

$$
f=f_{s} \frac{c}{c-V \cos \left[\tan ^{-1}\left(\frac{h}{V_{t}+x_{0}}\right)\right]}
$$

The velocity of an arrow can be calculated differently by types of arrows and microphone. The Doppler-shifted method using sound can calculate on a slower then speed of sound. And must calculate in a sound-free environment because another sound can generate high noise that can make the calculation missed. Another way of using Doppler shifted by using laser radar. By using millimeter wave radar system it can calculate speed an range using capture the signal of laser (Radar, 2011).

Figure 4 show the schematic of Doppler laser radar for vice. Using narrow laser width to improve the precision of measuring the speed. It divides into 2 optical splitters. One branch is used for the signal when another transits to external modulator. The received laser light split into 2 parts: one part is sent to a Photo Diode (PD) PD2 and the other part assigned to a coupler. The laser light sent to PD2 is converted into an electrical signal and is used to obtain the target range information. The laser sent to the coupler interferes with the reference signal and send to PD1. PD1 outputs the beat frequency of the received and reference signals. Fourier analysis is used to obtain the Doppler frequency and thus acquire the target speed information. All fibers are maintaining the polarization to keep the polarization state of the light signal constant.

High speed video recording: Using a high-speed camera to record the velocity of arrow become another way because it's more reliable and cannot disturb the flight of 


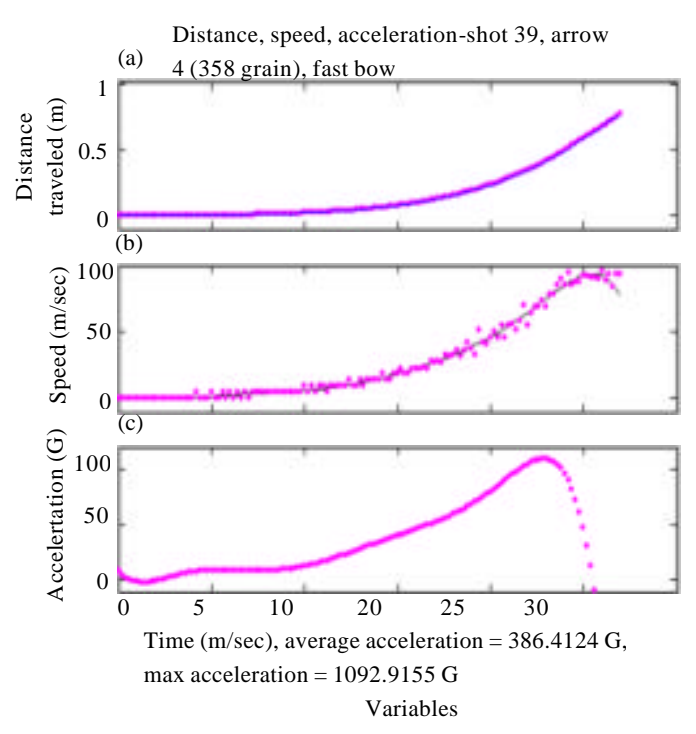

Fig. 5: Arrow distance, speed and acceleration in axial direction during launch with compound bow (Barton et al., 2011)

an arrow. Using high-speed camera record, we can estimate the trajectory by processing the video. There is some disadvantage using a high-speed camera such as the camera itself can cost more than $\$ 2000$. The view angle of a camera must be a perfect position, so, trajectory of an arrow can be calculated precisely (Barton et al., 2011).

Figure 5 with an example using a compound bow, to calculate for travel of flight is using 2 parameters, first using acceleration estimate full range of compound. And the second one is using time of flight. With these value, we can calculate the speed of an arrow.

Laser based system: Using a laser-based system to measure the speed of arrow become the good one because using laser can determine speed even at a supersonic rate (Lu et al., 1993). We can use a laser to measure the speed of an arrow which place on all edge of arrow trajectory. That system called Target Measurement System (TMS) (Baek et al., 2012).

Figure 6 the concept of laser-based usually using laser receiver and transmitter, the receiver will transform light flux from the receiver into an electronic signal. When arrow or projectile passes through a laser, the light will be disturb and make the signal will be weak. The weak signal will be amplified and sampled to calculate the speed of arrow or projectile (Baek et al., 2012; Liu and Yu, 2009; Zhao et al., 2013).

IMU sensor: IMU sensor has been using from 1930 it had used in aircraft navigation and it's massive because it

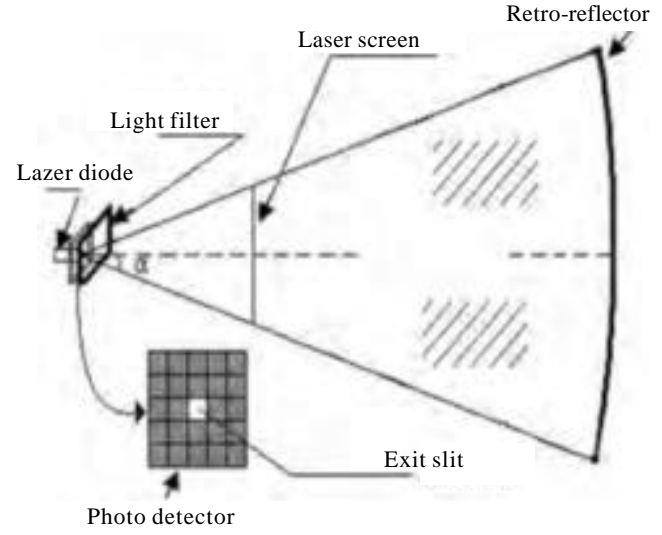

Fig. 6: Optical system schematic (Liu and Yu, 2009)

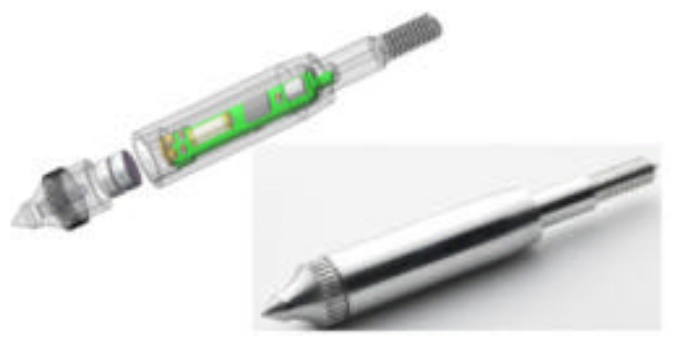

Fig. 7: IMU sensor on arrow

contains some sensors and needs a significant power source to run it (Ahmad et al., 2013). IMU sensor usually consists of three sensors, an accelerometer to measure the speed, gyroscope to measure angular rotation and magnetometer to measure point of a compass.

With modern technology, IMU sensor has improved using Micro-Electro Mechanical System (MEMS) (Ahmad et al., 2013; Kevin et al., 2011; Yuan et al., 2013). With a small sensor, IMU can implement in a various order such as in baseball, arrow, bowling or quadcopter (McGinnis and Perkins, 2012).

Figure 7 is one of the examples of implementation IMU sensor on an arrow for measuring velocity or trajectory of the arrow. The concept is to make sensor inside the arrow; the speed will be more accurate because the sensor speed will be same as arrow's speed. Sometimes IMU sensor also using GPS to make more precise precisely to measure a speed of an arrow (Yoon and Peng, 2014).

But there is some disadvantage using of IMU sensor such as the sensor is fragile because some component is made of plastic. The sensor can make unbalance in particular application such as an arrow when sensor places it in the tip of an arrow, make the arrow disturb because of the front weight have more mass than back (Barton et al., 2011, 2012). 


\section{RESULTS AND DISCUSSION}

A chronograph is standard for measuring a type of objects such as bullets, arrow or any projectile. There is 2 type of chronograph model that have been invented, the optical chronograph and chronograph watch. Optical chronograph is more expensive than another method to measure the speed of an arrow. But there is some disadvantage such as the cost of each unit can cost up to $\$ 900$ and the device must be placed in a bright area because the device is light sensitive.

With acoustic is one of the alternative ways to measure the speed of arrow by using the sound of arrow's turbulence. It's the cheapest way to calculate but there is disadvantage such as the area must noise-free because the microphone needs to get better sound without any noise.

High-speed video recording can also to measure the speed of arrow by capturing the video of arrow's movement. And processing with an algorithm to calculate speed and trajectory of the arrow. But the main disadvantage in this method is the camera price is too way expensive it cost more than $\$ 2000$ for each camera and the camera needs to place in the right angle to get all information data in arrow to calculate into speed.

And laser system is reliable to measure the speed of arrow because using laser can determine speed even at supersonic speed (Lu et al., 1993). We can use a laser to measure the speed of an arrow that place on all edge of arrow trajectory. That system called Target Measurement System (TMS). But there is disadvantage such as an area of the sensor need to be clear from any object because the laser can capture an even small object that passes through into the sensor.

And IMU sensor is one of the latest technology that to measure the speed of an arrow by usually consists of three sensors, there is an accelerometer to measure the speed, a gyroscope to measure angular rotation and a magnetometer to measure point of a compass. With all sensor that implant into the device, the measurement of an arrow become more accurate because the sensor travels together with the arrow. The disadvantage of using IMU sensor is the sensor need the battery to run in and need wireless to send the data to get the actual speed of the sensor.

\section{CONCLUSION}

Based on the tests that have achieved against some of the methods, then it can be taken some conclusions as follows: In archery, an archer needs accuracy in aiming the target and can predict the speed of an arrow because of archer's paradox. There are some ways of measuring a velocity of an arrow such as using chronograph, acoustic Doppler shifter and high-speed video recording, laser-based system and IMU sensor. The best way to measurement velocity of the arrow by using IMU sensor because of the speed of sensor as same as the speed of an arrow

\section{REFERENCES}

Ahmad, N., R.A.R. Ghazilla, N.M. Khairi and V. Kasi, 2013. Reviews on various Inertial Measurement Unit (IMU) sensor applications. Intl. J. Signal Process. Syst., 1: 256-262.

Backwell, L., F. d'Errico and L. Wadley, 2008. Middle stone age bone tools from the Howiesons Poort layers, Sibudu Cave, South Africa. J. Archaeol. Sci., 35: 1566-1580.

Baek, G.D., S.P. Cheon, I.S. Lee and S.S. Kim, 2012. Parameter calibration of laser scan camera for measuring the impact point of arrow. J. Korean Soc. Manuf. Technol. Eng., 21: 76-84.

Barton, J., J. Vcelak, J. Torres-Sanchez, B. O'Flynn and C. O'Mathuna et al., 2011. A miniaturised arrow ballistic measurement system. Proceedings of the IEEE 2011 International Conference on Sensors, October 28-31, 2011, IEEE, Limerick, Ireland, ISBN:9781-4244-9290-9, pp: 1289-1292.

Barton, J., J. Vcelak, J. Torres-Sanchez, B. O'Flynn and C. O'Mathuna et al., 2012. Arrow-mounted ballistic system for measuring performance of arrows equipped with hunting broadheads. Procedia Eng., 34 : 455-460.

Brett, 2016. How to use a chronograph and tachymeter on a wristwatch. UrbanDaddy Inc., New York, USA. https://www.artofmanliness.com/2016/11/22/usechronograph-tachymeter-wristwatch/

French, M. and T. Kirk, 2007. Measuring the flight of an arrow using the acoustic Doppler shift. Mech. Syst. Signal Process., 21: 1188-1191.

Kevin, K., N.C. Perkins, H. Churchill, R. McGinnis, R. Doss and R. Hickland, 2011. Bowling ball dynamics revealed by miniature wireless MEMS inertial measurement unit. Sports Eng., 13: 95-104.

Liu, J. and L. Yu, 2009. Laser-based apparatus for measuring projectile velocity. Proceedings of the 9th International Conference on Electronic Measurement and Instruments ICEMI'09, August 16-19, 2009, IEEE, Beijing, China, ISBN:978-1-42443863-1, pp: 595-598. 
Lu, S.T., C. Chou, M.C. Lee and Y.P. Wu, 1993. Electro-optical target system for position and speed measurement. IEE. Proceedings A. Sci. Meas. Technol., 140: 252-256.

McGinnis, R.S. and N.C. Perkins, 2012. A highly miniaturized, wireless inertial measurement unit for characterizing the dynamics of pitched baseballs and softballs. Sensors, 12: 11933-11945.

Radar, A.L., 2011. Short papers speed measurement in car applications. IEEE. Trans. Intell. Transp. Syst., 13: 1-6.

Tao, J.H., 2006. The general method for fixing the gauges of relativistic astronomical reference systems. Astrophys. Space Sci., 302: 93-98.

Vroom, G.B., 1923. The langley chronograph. J. Am. Soc. Nav. Eng., 35: 375-380.
Yoon, J.H. and H. Peng, 2014. A cost-effective sideslip estimation method using velocity measurements from two GPS receivers. IEEE. Trans. Veh. Technol., 63: 2589-2599.

Yuan, Q., I.M. Chen and A. Caus, 2013. Human velocity tracking and localization using $3 \mathrm{IMU}$ sensors. Proceedings of the 2013 6th IEEE International Conference on Robotics, Automation and Mechatronics (RAM), November 12-15, 2013, IEEE, Manila, Philippines, ISBN:978-1-4799-1201-8, pp: 25-30.

Zhao, D., H. Zhou, J. Liu, B. Zhang and Q. Luo, 2013. High-precision velocity measuring system for projectiles based on retroreflective laser screen. Optik Intl. J. Light Electron Opt., 124: 544-548. 